\title{
Particle production from axial fields
}

\author{
Antonio L. Maroto* \\ Astronomy Centre, University of Sussex, Falmer, Brighton, United Kingdom BN1 9QJ
}

(Received 21 October 1998; published 2 February 1999)

\begin{abstract}
We study the production of massive fermions in arbitrary vector and axial-vector classical backgrounds using effective action techniques. A perturbative calculation shows the different features of each field and in particular it is seen that pure temporal axial fields can produce particles whereas it is not possible for a pure vector background. We also analyze from a non-perturbative point of view a particular configuration with constant electric and axial fields and show that the presence of the axial background inhibits the production from the electric field. [S0556-2821(99)04504-X]

PACS number(s): 98.80.Cq, 04.62.+v
\end{abstract}

\section{INTRODUCTION}

The production of particles from classical backgrounds has become a very active area of research in the past years. We can find it in numerous and disconnected fields of physics such as cosmology [1,2], heavy-ion collisions or even plasma physics [3]. The pioneer work of Schwinger [4] mainly focused on the production of electron-positron pairs by strong electrostatic fields. Since then, many other sources of particles creation have been studied in the literature. Thus for example we can mention time dependent gravitational fields [2], varying Planck mass models [5], compactification of extra dimensions [6], dilaton field [5], inflaton field, etc. The original Schwinger's work was based on the proper-time technique for the evaluation of the effective action. This method allowed him to obtain an exact non-perturbative result for the total number of particles produced. However, although some other electromagnetic configurations have been studied [7] and some particular cases have also been solved [8], in general the number of models for which exact results can be obtained is very limited.

In spite of its generalized use, it is probably in cosmology where particle production has been applied more extensively. Thus, for instance, it is believed that the presence of any small anisotropy in the early universe could have been erased very effectively by particle emssion processes [9]. The theory of reheating after inflation is also based on the resonant production of particles due to the oscillations of the inflaton field [10]. The reheating of fermions has been considered in [11]. In addition, the formation of large scale structures in the early universe is closely related to the generation from vacuum fluctuation of small seed density inhomogeneities that due to the gravitational instability grew to give rise to the current galactic structure. The generation of the vacuum fluctuations can be studied in much the same way as the production of scalar particles in a RobertsonWalker background [12]. Recently the problem of particle production has also been extended to the area of string cosmology in which, apart from the gravitational background, there is also an additional scalar field, the dilaton, that can also give rise to the generation of particles $[5,13]$.

*Email address: alm@star.cpes.susx.ac.uk
In general, the problem of calculating particle production can be approached in two different ways: on the one hand, the Bogolyubov technique [1], which allows the calculation of the spectrum of the particle produced. It is based on the resolution of the harmonic-oscillator equation with variable frequency and only in some particular cases permits the derivation of exact results. On the other hand, we have the already mentioned effective action technique, which allows one to obtain the total number of particles produced in a much simpler way $[4,14,15]$, although the difficulties in finding exact results are also present.

Most of the existing works about the creation of particles from vacuum fluctuations concentrate on the production of boson fields in the presence of scalar, vector or gravitational backgrounds. In this paper we will study a different source for the production of fermions: it is the presence of general vector and axial-vector backgrounds. We will thus extend the Schwinger's work by including the effects of a nonvanishing axial field. Classical axial backgrounds appear naturally in modern theories of gravity such as supergravity [16] or in low-energy string effective actions [17,18]. Both theories contain torsion (or axion) fields as a fundamental ingredient and, in fact, recently several solutions with nonvanishing axial fields have been found in the context of string cosmology $[19,20]$. On the other hand, the idea of modifying general relativity by introducing an arbitrary metric connection with torsion is an old one [21], and in some sense quite natural from the point of view of the gauge theories of gravity [22]. This torsion field is coupled minimally to fermions by means of its pseudotrace, thus providing a new mechanism for the production of particles. In fact in these gravitational theories, torsion would be the dominant mechanism for the production of massless fermions in cosmological Robertson-Walker backgrounds. This is due to the fact that when gravity is minimally coupled to massless fermions, the theory is conformally invariant. This implies the wellknown result of absence of particle production. However, the presence of additional fields, such as torsion or metric anisotropies, breaks that invariance. Our work will be based on the effective action method, first from a perturbative point of view, and then we will study a particular case in which a non-perturbative calculation is viable.

The paper is organized as follows. In Sec. II we introduce the Lagrangian for the model and also give a brief introduc- 
tion to the effective action technique. In Sec. III, we perform the perturbative calculation and compare the result with the pure vector case. Section IV is devoted to the particular case with constant electric and axial field and a non-perturbative result is obtained in the limit of small axial fields and in Sec. $\mathrm{V}$ we give the main conclusions of the work. Finally we have included an appendix with some useful formulas of standard perturbation theory in quantum mechanics.

\section{MODEL LAGRANGIAN AND THE EFFECTIVE ACTION METHOD}

We will consider the following interaction Lagrangian for massive fermions minimally coupled to Abelian vector and axial-vector fields. For simplicity we will use the left-right notation and at the end we will recover the vector-axial fields:

$$
\mathcal{L}=\bar{\psi}(i \not D-m+i \epsilon) \psi
$$

where $i \not D D=i \gamma^{\mu}\left(\partial_{\mu}+i A_{\mu} P_{L}+i B_{\mu} P_{R}\right)$. As usual the $+i \epsilon$ factor is introduced to ensure the convergence of the path integral and the left and right projectors are defined as $P_{L}$ $=\left(1-\gamma_{5}\right) / 2$ and $P_{R}=\left(1+\gamma_{5}\right) / 2$. We will use the chiral representation for the Dirac matrices in which $\gamma_{5}$ is diagonal. The coupling constants are included in the own fields.

Let us now introduce the effective action (EA) for the $A_{\mu}$ and $B_{\mu}$ fields that is obtained after integrating the fermions out:

$$
\begin{aligned}
\langle 0, t \rightarrow \infty \mid 0, t \rightarrow-\infty\rangle & =Z[A, B]=e^{i W[A, B]} \\
& =N \int d \psi d \bar{\psi} \exp \left(i \int d^{4} x \mathcal{L}\right) \\
& =N \operatorname{det}(i \not D-m+i \epsilon) .
\end{aligned}
$$

Here, $|0, t \rightarrow \pm \infty\rangle$ denote the initial and final vacuum states that in general will be different due to the presence of the external sources. $N$ is a normalization constant that is taken as usual in such a way that $Z[0,0]=1$; this will allow us to discard the vacuum divergences as shown below.

The EA will be in general a complex non-local functional in the external fields. Its real part will contain the divergences that will be renormalized by adding suitable local counterterms to the action. The imaginary part will be finite and will contain information about the particle production probabilities. In fact, the probability that the vacuum remains stable is given by $|\langle 0, t \rightarrow \infty \mid 0, t \rightarrow-\infty\rangle|^{2}$. Therefore the probability that the vacuum decays by particle emission will be given by

$$
\begin{aligned}
P & =1-|\langle 0, t \rightarrow \infty \mid 0, t \rightarrow-\infty\rangle|^{2} \\
& =1-e^{-2 \operatorname{Im} W[A, B]} \simeq 2 \operatorname{Im} W[A, B] .
\end{aligned}
$$

When we only have a vector field such that its corresponding electric field is constant, the probability density per unit time and unit volume $p$ can be obtained exactly and the result is given by [4]

$$
p=\frac{e^{2} E^{2}}{4 \pi^{3}} \sum_{n=1}^{\infty} \frac{1}{n^{2}} e^{-m^{2} n \pi / e E} .
$$

However, for non-constant electromagnetic fields or when the axial part is switched on, the computation becomes very involved and it is necessary to rely on some perturbative method.

Before concluding this section we will mention that although the notion of torsion appears in strings and supergravity in slightly different ways, both can be interpreted as the antisymmetric part of the affine connection in pseudoRiemannian geometry [18]. Thus, if the components of the metric connection are $\hat{\Gamma}_{\mu \nu}^{\lambda}$, its antisymmetric part $T_{\mu \nu}^{\lambda}$ $=\hat{\Gamma}_{\mu \nu}^{\lambda}-\hat{\Gamma}_{\nu \mu}^{\lambda}$ is known as the torsion tensor. By means of the Einstein equivalence principle, it is now possible to minimally couple torsion to fermion fields $[23,24]$; one gets

$$
\mathcal{L}=\bar{\psi} i \gamma^{\mu}\left(\partial_{\mu}+\Omega_{\mu}+\frac{i}{8} S_{\mu} \gamma_{5}\right) \psi
$$

where $S_{\rho}=\epsilon_{\mu \nu \lambda \rho} T^{\mu \nu \lambda}$ is the torsion pseudotrace and $\Omega_{\mu}$ the spin connection.

\section{PERTURBATIVE METHOD}

In this section we present the evaluation of the EA in Eq. (2) as an expansion in the external fields, i.e. an expansion in coupling constants. Let us start by writing

$$
W[A, B]=i \operatorname{Tr} \log (i \not D-m+i \epsilon)
$$

which can formally be expanded as

$$
W[A, B]=i \sum_{k=1} \frac{(-1)^{k}}{k} \operatorname{Tr}\left[(i \not b-m)^{-1}\left(A P_{L}+\not B P P_{R}\right)\right]^{k}
$$

where the Dirac propagator is defined as usual by

$$
(i b-m)_{x y}^{-1}=\int d \tilde{q} e^{-i q(x-y)} \frac{q+m}{q^{2}-m^{2}+i \epsilon} .
$$

The functional traces $\operatorname{Tr}$ are evaluated in dimensional regularization with $D=4-\epsilon$ and $d \tilde{q}=\mu^{\epsilon} d^{D} q /(2 \pi)^{D}$. The lowest order contribution in the expansion is given by the twopoint terms, i.e.

$$
\begin{aligned}
W[A, B]^{(2)}= & \frac{i}{2} \int d^{4} x d^{4} y d \tilde{p} d \tilde{q} \frac{\not{q}+m}{q^{2}-m^{2}} \\
& \times e^{-i q(x-y)}\left(A_{y} P_{L}+\boldsymbol{B}_{y} P_{R}\right) \frac{\not p+m}{p^{2}-m^{2}} \\
& \times e^{-i p(y-x)}\left(A_{x} P_{L}+B_{x} P_{R}\right) .
\end{aligned}
$$

Expanding these terms and defining $k=q-p$, we obtain the following expression: 


$$
\begin{aligned}
W[A, B]^{(2)}= & 2 i \int d^{4} x d^{4} y d \tilde{k} d \tilde{q} \frac{e^{-i k(x-y)}}{\left(q^{2}-m^{2}\right)\left[(q-k)^{2}-m^{2}\right]} \\
& \times\left(q^{\mu} A_{\mu}^{y} \frac{(q-k)^{\rho}}{2} A_{\rho}^{x}-q^{\mu} \frac{(q-k)}{2} A_{y}^{\nu} A_{\nu}^{x}\right. \\
& \left.+q^{\mu} A_{\mu}^{x} A_{\nu}^{y} \frac{(q-k)^{\nu}}{2}+\frac{m^{2}}{2} g^{\mu \nu} A_{\mu}^{y} B_{\nu}^{x}+(A \rightarrow B)\right) .
\end{aligned}
$$

All the integrals, except for that involved in the term proportional to $\mathrm{m}^{2}$, can now be reduced to a common form that is evaluated in dimensional regularization:

$$
\begin{aligned}
\int d \tilde{q} \frac{q^{\alpha}(q-k)^{\beta}}{\left(q^{2}-m^{2}\right)\left[(q-k)^{2}-m^{2}\right]} \\
=\frac{i}{(4 \pi)^{D / 2}}\left(k^{\alpha} k^{\beta} \Gamma(2-D / 2) \int_{0}^{1} d t \frac{t^{2}-t}{\mathcal{D}^{2-D / 2}}\right. \\
\left.\quad-\frac{g^{\alpha \beta}}{2} \Gamma(1-D / 2) \int_{0}^{1} d t \mathcal{D}^{D / 2-1}\right)
\end{aligned}
$$

with $\mathcal{D}=m^{2}-k^{2} t(1-t)$. The integral proportional to $m^{2}$ is nothing but

$$
\begin{aligned}
& \int d \tilde{q} \frac{1}{\left(q^{2}-m^{2}\right)\left[(q-k)^{2}-m^{2}\right]} \\
& =\frac{1}{(4 \pi)^{D / 2}} \Gamma(2-D / 2) \int_{0}^{1} d t \mathcal{D}^{D / 2-2}
\end{aligned}
$$

The imaginary part of $W[A, B]$ can be easily extracted from the integrals in the Feynman parameter $t$. They give rise to

$$
\begin{aligned}
& \operatorname{Im} \int_{0}^{1} d t\left(t^{2}-t\right) \log \left[m^{2}-k^{2} t(1-t)-i \epsilon\right] \\
& =\int_{0}^{1} d t\left(t^{2}-t\right) \times\left\{\begin{array}{cc}
0, & m^{2}>k^{2} t(1-t), \\
-\pi / 2, & m^{2}=k^{2} t(1-t), \\
-\pi, & m^{2}<k^{2} t(1-t)
\end{array}\right. \\
& =\frac{\pi}{4} \sqrt{1-\frac{4 m^{2}}{k^{2}}}\left(\frac{2}{3}+\frac{4 m^{2}}{3 k^{2}}\right), \quad \frac{k^{2}}{m^{2}}>4,
\end{aligned}
$$

and in a similar fashion we obtain

$$
\begin{aligned}
& \operatorname{Im} \int_{0}^{1} d t\left[m^{2}-k^{2} t(1-t)\right] \log \left[m^{2}-k^{2} t(1-t)-i \epsilon\right] \\
& \quad=-\pi \sqrt{1-\frac{4 m^{2}}{k^{2}}}\left(\frac{2}{3} m^{2}-\frac{k^{2}}{6}\right)
\end{aligned}
$$

and

$$
\begin{aligned}
& \operatorname{Im} \int_{0}^{1} d t \log \left[m^{2}-k^{2} t(1-t)-i \epsilon\right] \\
& \quad=-\pi \sqrt{1-\frac{4 m^{2}}{k^{2}}}\left(\frac{2}{3} m^{2}-\frac{k^{2}}{6}\right), \quad \frac{k^{2}}{m^{2}}>4
\end{aligned}
$$

Putting all the contributions together and changing to the vector and axial-vector fields defined by $V=B+A$ and $S$ $=B-A$ respectively, we obtain the final result for the imaginary part in terms of the Fourier transformed fields:

$$
\begin{aligned}
\operatorname{Im} W^{(2)}[A, B]= & \frac{1}{8 \pi^{2}} \int d \tilde{k} \theta\left(k^{2}-4 m^{2}\right) \sqrt{1-\frac{4 m^{2}}{k^{2}}} \\
& \times\left[-\frac{1}{6}\left(1+\frac{2 m^{2}}{k^{2}}\right)\left[F_{\mu \nu}(k) F^{\mu \nu}(-k)\right.\right. \\
& \left.\left.+S_{\mu \nu}(k) S^{\mu \nu}(-k)\right]+2 m^{2} S_{\mu}(k) S^{\mu}(-k)\right] .
\end{aligned}
$$

We see that unlike the vector case, the axial contribution to the imaginary part has an additional term proportional to $m^{2} S^{2}$. This term is prohibited by gauge invariance in the vector case; however, as it is well-known it may appear in axial theories with massive fermions since those theories violate the corresponding gauge invariance. In fact studying the divergences that appear in the model we see that they are proportional to the following operators: $F_{\mu \nu} F^{\mu \nu}, S_{\mu \nu} S^{\mu \nu}$ and $m^{2} S_{\mu} S^{\mu}[25,24]$. The $S^{4}$ operator, although having the same dimension, does not contribute to the divergent part. Therefore it is only neccesary to introduce a kinetic and a mass counterterms for the axial field in order to render the theory finite.

Since the integrand of imaginary part has to be understood as a probability density, it is important to verify that it is always positive. As far as $k^{2}>4 m^{2}$, because of the step function present in Eq. (16), it is possible to find a reference frame in which $\vec{k}=0$. Then we have

$$
\begin{aligned}
\frac{1}{3}(1 & \left.+\frac{2 m^{2}}{k_{0}^{2}}\right)\left[k_{0} S^{0}(k) k_{0} S^{0}(-k)-k_{0}^{2} S_{\mu}(k) S^{\mu}(-k)+S \rightarrow V\right] \\
& +2 m^{2} S_{\mu}(k) S^{\mu}(-k) \\
= & \frac{1}{3}\left(1+2 \frac{m^{2}}{k_{0}^{2}}\right)\left(\left|S_{i}\right|^{2}+\left|V_{i}\right|^{2}\right)+2 m^{2}\left(\left|S_{0}\right|^{2}-\left|S_{i}\right|^{2}\right) \\
= & \frac{1}{3}\left(k_{0}^{2}-4 m^{2}\right)\left|S_{i}\right|^{2}+\frac{1}{3}\left(k_{0}^{2}+2 m^{2}\right)\left|V_{i}\right|^{2}+2 m^{2}\left|S_{0}\right|^{2} \geqslant 0 .
\end{aligned}
$$

From this expression we can extract some of the different features of the production from vector and axial fields. First we see that if the axial field is purely spatial in the above reference frame, i.e., $S_{0}=0$, and we choose $S_{i}(k)=V_{i}(k)$, 
then the production from pure axial fields is always supressed with respect to the pure vector case. However, when $S_{0}(k)=V_{0}(k)$ and $S_{i}=V_{i}=0$ then, whereas there is no production in the vector case, it is possible to create particles in the axial one. In the massless case both fields give rise to the same amount of particles.

\section{CONSTANT ELECTRIC AND AXIAL FIELDS: A NON-PERTURBATIVE RESULT}

In the previous section we have obtained the particle production probabilities up to second order in perturbation theory. This is in general a good approximation for small background fields; however, even in those cases, it does not contain all the information about the particle production processes. In this section we will study a particular configuration of vector and axial fields for which it is possible to find an expression for the imaginary part which is nonperturbative in the electric field, in the limit $S^{2} \ll E$ and $m^{2} S^{2} / E^{2}<1$.

Let us start by introducing the operators $X_{\mu}$ and $P_{\mu}$ acting on states $|x\rangle$ and $|p\rangle$ in the usual form: $X_{\mu}|x\rangle=x_{\mu}|x\rangle$ and $P_{\mu}|p\rangle=p_{\mu}|p\rangle$. In addition $\left\langle x\left|P_{\mu}\right| \phi\right\rangle=i \partial_{\mu}\langle x \mid \phi\rangle$. The comutator is given by $\left[X_{\mu}, P_{\nu}\right]=-i g_{\mu \nu}$ and $\langle p \mid x\rangle$ $=e^{i p x} /(2 \pi)^{2}$.

Following Itzykson and Zuber [26] we recast the Dirac operator in Eq. (1) as

$$
i \not D=\left(\not P-A P_{L}-\not B P_{R}\right)
$$

and taking the transpose we have

$$
\left(\boldsymbol{P}-\not A P_{L}-\not B P_{R}\right)^{t}=-C\left(\boldsymbol{P}-\not A P_{R}-\not B P_{L}\right) C^{-1}
$$

where $C=i \gamma^{2} \gamma^{0}$ is the charge conjugation matrix that satisfies $C \gamma_{\mu} C^{-1}=-\gamma_{\mu}^{t}$ and $C \gamma_{5} C^{-1}=\gamma_{5}^{t}$. The effective action in Eq. (2) can be written with this notation as

$W[A, B]=-i \operatorname{Tr} \log \left(\left(\boldsymbol{P}-\mathbb{A} P_{L}-\boldsymbol{B} P_{R}-m+i \boldsymbol{\epsilon}\right) \frac{1}{\boldsymbol{P}-m+i \boldsymbol{\epsilon}}\right)$

where we have explicitly introduced the normalization factor $N$ in the last term. The effective action can also be written in terms of the transposed operators

$$
\begin{aligned}
W[A, B]= & -i \operatorname{Tr} \log \left(\left(\boldsymbol{P}-\mathbb{A} P_{L}-\not B P_{R}-m+i \boldsymbol{\epsilon}\right)^{t}\right. \\
& \left.\times\left(\frac{1}{\boldsymbol{P}-m+i \boldsymbol{\epsilon}}\right)^{t}\right) \\
= & -i \operatorname{Tr} \log \left(\left(\boldsymbol{P}-A P_{R}-\not B P_{L}+m-i \boldsymbol{\epsilon}\right)\right. \\
& \left.\times \frac{1}{\boldsymbol{P}+m-i \boldsymbol{\epsilon}}\right) .
\end{aligned}
$$

Adding both expressions we get

$$
\begin{aligned}
& 2 W[A, B]=-i \operatorname{Tr} \log \left(\left(\boldsymbol{P}-\mathbb{A} P_{L}-\not B P_{R}-m+i \boldsymbol{\epsilon}\right)\right. \\
& \left.\times\left(\boldsymbol{P}-\boldsymbol{A} P_{R}-\boldsymbol{B} P_{L}+m-i \boldsymbol{\epsilon}\right) \frac{1}{P^{2}-m^{2}+i \boldsymbol{\epsilon}}\right) \\
& =-i \operatorname{Tr} \log \left[\left(\left(P_{\mu}-A_{\mu} P_{R}-B_{\mu} P_{L}\right)^{2}-\frac{i}{4}\left(A_{\mu \nu} P_{R}\right.\right.\right. \\
& \left.\left.+B_{\mu \nu} P_{L}\right)\left[\gamma^{\mu}, \gamma^{\nu}\right]+m(\mathbb{A}-\mathbb{B}) \gamma_{5}-m^{2}+i \epsilon\right) \\
& \left.\times \frac{1}{P^{2}-m^{2}+i \epsilon}\right] \text {. }
\end{aligned}
$$

Finally we change to the vector and axial fields; the effective action is then written as

$$
\begin{aligned}
2 W[V, S]= & -i \operatorname{Tr} \log \left[\left(\left(P_{\mu}-V_{\mu}-S_{\mu} \gamma_{5}\right)^{2}\right.\right. \\
& \left.-\frac{i}{4}\left(V_{\mu \nu}+S_{\mu \nu} \gamma_{5}\right)\left[\gamma^{\mu}, \gamma^{\nu}\right]-m \$ \gamma_{5}-m^{2}+i \epsilon\right) \\
& \left.\times \frac{1}{P^{2}-m^{2}+i \epsilon}\right] .
\end{aligned}
$$

Here $V_{\mu \nu}=\partial_{\mu} V_{\nu}-\partial_{\nu} V_{\mu}$ and $S_{\mu \nu}=\partial_{\mu} S_{\nu}-\partial_{\nu} S_{\mu}$. Let us take the following background fields: $V^{\mu}=\left(0,0,0, B x^{1}\right)$ and $S^{\mu}$ $=(0,0,0, S)$ with $B$ and $S$ arbitrary constants. This choice corresponds to a constant magnetic field $B$ along the $y$ axis and a constant axial field $S$ in the $z$ direction. Obviously the same result will be obtained if we choose the fields in different spatial directions, provided they are orthogonal. By means of the Schwinger proper-time integral we can write

$2 W[V, S]$

$$
\begin{aligned}
= & -i \operatorname{Tr} \int_{0}^{\infty} \frac{d s}{s} e^{-i s\left(m^{2}-i \epsilon\right)} \\
& \times\left(\left\langle x\left|e^{i s\left(P_{0}^{2}-P_{1}^{2}-P_{2}^{2}-\left(P^{3}-B X^{1}-S \gamma_{5}\right)^{2}+i B\left[\gamma^{1}, \gamma^{3}\right] / 2-m S \gamma_{3} \gamma_{5}\right)}\right| x\right\rangle\right. \\
& \left.-\left\langle x\left|e^{i s P^{2}}\right| x\right\rangle\right) .
\end{aligned}
$$

The action of the translation operator will simplify this expression:

$$
\left(P^{3}-B X^{1}-S \gamma_{5}\right)^{2}=e^{-i\left(P^{1} P^{3} / B\right)}\left(-B X^{1}-S \gamma_{5}\right)^{2} e^{i\left(P^{1} P^{3} / B\right)} .
$$

Let us now introduce complete sets of momentum eigenstates: 
$2 W[V, S]$

$$
\begin{aligned}
= & -i \operatorname{Tr} \int_{0}^{\infty} \frac{d s}{S} e^{-i s\left(m^{2}-i \epsilon\right)} \\
& \times\left[\left(\frac{1}{(2 \pi)^{4}} \int d^{4} p d \tilde{p}^{1} e^{i s\left(p_{0}^{2}-p_{2}^{2}\right)} e^{i p^{3}\left(\tilde{p}^{1}-p^{1}\right) / B} e^{-i\left(\tilde{p}^{1}-p^{1}\right) x^{1}}\right.\right. \\
& \left.\times\left\langle p^{1}\left|e^{i s\left(-P_{1}^{2}-\left(-B X^{1}-S \gamma_{5}\right)^{2}+i B\left[\gamma^{1}, \gamma^{3}\right] / 2-m S \gamma_{3} \gamma_{5}\right)}\right| \tilde{p}^{1}\right\rangle\right) \\
& \left.-\int \frac{d^{4} p d^{4} \tilde{p}}{(2 \pi)^{4}} e^{i(\tilde{p}-p) x}\left\langle p\left|e^{i s P^{2}}\right| \tilde{p}\right\rangle\right]
\end{aligned}
$$

Performing the integral in the $p^{3}$ variable and in the $p^{0}$ and $p^{2}$ by means of

$$
\int_{-\infty}^{\infty} d q e^{-i s q^{2}}=\sqrt{\frac{\pi}{i s}}
$$

the above expression reduces to:

$$
2 W[V, S]
$$

$$
\begin{aligned}
= & i \operatorname{Tr} \int_{0}^{\infty} \frac{d s}{s} e^{-i s\left(m^{2}-i \epsilon\right)} \\
& \times\left[\frac{B}{8 \pi^{2}} \int d p\left\langle p\left|e^{i s\left(-P^{2}-\left(B X+S \gamma_{5}\right)^{2}+i B\left[\gamma^{1}, \gamma^{3}\right] / 2-m S \gamma_{3} \gamma_{5}\right)}\right| p\right\rangle\right. \\
& \left.-\frac{i}{(4 \pi)^{2} s^{2}}\right]
\end{aligned}
$$

where for simplicity we have denoted $X=X^{1}$ and $P=P_{1}$. The integral in $p$ together with the matrix trace can be considered as the trace of the evolution operator corresponding to the Hamiltonian $H=H_{0}+H_{1}$ in ordinary quantum mechanics with

$$
H_{0}=\left(\begin{array}{cc}
-P^{2}-(B X+S)^{2}-B \sigma^{2} & 0 \\
0 & -P^{2}-(B X-S)^{2}-B \sigma^{2}
\end{array}\right)
$$

and

$$
H_{1}=\left(\begin{array}{cc}
0 & m S \sigma^{3} \\
m S \sigma^{3} & 0
\end{array}\right)
$$

with $\sigma^{i}$ the corresponding Pauli matrices. The problem of evaluating the effective action is thus reduced to the calculation of the spectrum of the $H$ operator. However, since we cannot obtain such spectrum in an exact form, we will consider $H_{1}$ as a small perturbation. With that purpose, we will assume that the contributions to the spectrum coming from the perturbation are smaller than the eigenvalues of $H_{0}$, i.e., $m^{2} S^{2}<B^{2}$. We can then apply the standard Kato theory for time-independent perturbations in quantum mechanics [27].
The $H_{0}$ operator is made out of two shifted harmonicoscillator Hamiltonians with mass $M=1 / 2$, frequency $\omega$ $=2 B$ and a new coupling to the magnetic field. Its spectrum $\left\{\lambda_{n, i}^{(0)}\right\}$ and eigenfunctions $\left\{\psi_{n, i}^{(0)}\right\}$ with $\{n=0, \ldots, \infty, i$ $=1, \ldots, 4\}$ can be easily obtained; they are given by

$$
\begin{aligned}
& \lambda_{n, 1}^{(0)}=E_{n}+B, \quad \psi_{n, 1}^{(0)}=\frac{1}{\sqrt{2}}\left(\begin{array}{c}
i \phi_{n} \\
\phi_{n} \\
0 \\
0
\end{array}\right), \\
& \lambda_{n, 2}^{(0)}=E_{n}-B, \quad \psi_{n, 2}^{(0)}=\frac{1}{\sqrt{2}}\left(\begin{array}{c}
\phi_{n} \\
i \phi_{n} \\
0 \\
0
\end{array}\right), \\
& \lambda_{n, 3}^{(0)}=E_{n}+B, \quad \psi_{n, 3}^{(0)}=\frac{1}{\sqrt{2}}\left(\begin{array}{c}
0 \\
0 \\
i \hat{\phi}_{n} \\
\hat{\phi}_{n}
\end{array}\right), \\
& \lambda_{n, 4}^{(0)}=E_{n}-B, \quad \psi_{n, 4}^{(0)}=\frac{1}{\sqrt{2}}\left(\begin{array}{c}
0 \\
0 \\
\hat{\phi}_{n} \\
i \hat{\phi}_{n}
\end{array}\right),
\end{aligned}
$$

where $E_{n}=-2 B(n+1 / 2)$ are the energy levels of the ordinary harmonic oscillator and

$$
\begin{aligned}
& \phi_{n}(x)=\sqrt{\frac{\sqrt{B}}{\sqrt{\pi} 2^{n} n !} H_{n}\left[\sqrt{B}\left(x+\frac{S}{B}\right)\right] e^{-B(x+S / B)^{2} / 2},} \\
& \hat{\phi}_{n}(x)=\sqrt{\frac{\sqrt{B}}{\sqrt{\pi} 2^{n} n !}} H_{n}\left[\sqrt{B}\left(x-\frac{S}{B}\right)\right] e^{-B(x-S / B)^{2} / 2}
\end{aligned}
$$

with $H_{n}$ the Hermite polynomials. Notice the different functional form of these two functions; it reflects the fact that the two harmonic oscillators in Eq. (29) are displaced in different ways. We will express the spectrum and the eigenfunctions of the complete Hamiltonian $H$ as perturbative series: $\lambda_{n, i}=\Sigma_{p=0} \lambda_{n, i}^{(p)}$ and $\psi_{n, i}=\Sigma_{p=0} \psi_{n, i}^{(p)}$. To first order in the perturbation, the spectrum is given by the solutions of the equation [27]

$$
\operatorname{det}\left|\left\langle\psi_{n, i}\left|H_{1}\right| \psi_{n, j}\right\rangle-\left(\lambda_{n, i}-\lambda_{n, i}^{(0)}\right) \delta_{i j}\right|=0 .
$$

The solutions imply $\lambda_{n, i}^{(1)}=0, \forall n$, $i$; i.e., there is no first order correction to the energies. The second order contributions are given by

$$
\lambda_{n, i}^{(2)}=\sum_{j \neq i} \frac{\left|\left\langle\psi_{n, i}^{(0)}\left|H_{1}\right| \psi_{n, j}^{(0)}\right\rangle\right|^{2}}{\lambda_{n, i}^{(0)}-\lambda_{n, j}^{(0)}} .
$$


Although, the $H_{0}$ spectrum is double degenerated, it is possible to use the above expression valid for non-degenerated spectra, since there is no contribution from states in the same multiplet. In any case, the explicit calculation using Kato theory yields the same results. The values of the second order perturbation can be evaluated in a straightforward way; we get

$$
\lambda_{n, 1}^{(2)}=\lambda_{n, 3}^{(2)}=\frac{m^{2} S^{2}}{2 B} \kappa_{n}^{2}, \quad \lambda_{n, 2}^{(2)}=\lambda_{n, 4}^{(2)}=-\frac{m^{2} S^{2}}{2 B} \kappa_{n}^{2}
$$

where

$$
\kappa_{n}=\left|\left\langle\phi_{n} \mid \hat{\phi}_{n}\right\rangle\right|=e^{-S^{2} / B}\left|L_{n}\left(2 \frac{S^{2}}{B}\right)\right|
$$

with $L_{n}$ the Laguerre polynomials. These polynomials are bounded as $n$ grows, in fact asymptotically we have [28] $L_{n}(x) \sim(1 / \sqrt{\pi}) e^{x / 2} x^{-1 / 4} n^{-1 / 4} \cos (2 \sqrt{n x}-\pi / 4)$ $+\mathcal{O}\left(n^{-3 / 4}\right)$ for $x>0$ and therefore $\kappa_{n}$ is bounded. In fact for $S^{2} / B \ll 1$, we can expand: $\kappa_{n}^{2}=1-\left(S^{2} / B\right)(4 n+2)$ $+\mathcal{O}\left(S^{4} / B^{2}\right)$. Therefore, as expected for small values of the axial field with respect to the magnetic one, $\kappa_{n}^{2}=1$ is a good approximation. Notice that, although the first correction grows like $n$, the growth must be controlled by higher order terms since the function is bounded. Let us stress that we have two different parameters in our problem: on the one hand, $S^{2} / B$, which we assume to be very small, and our perturbative parameter $m^{2} S^{2} /\left(2 B^{2}\right)$.

Using Eq. (A3) we can obtain the first correction to the eigenfunctions; we get

$$
\begin{aligned}
\left|\psi_{n, 1}^{(1)}\right\rangle & =\frac{i m S}{2 B} \kappa_{n}\left|\psi_{n, 4}^{(0)}\right\rangle,\left|\psi_{n, 2}^{(1)}\right\rangle=\frac{i m S}{2 B} \kappa_{n}\left|\psi_{n, 3}^{(0)}\right\rangle, \\
\left|\psi_{n, 3}^{(1)}\right\rangle & =\frac{i m S}{2 B} \kappa_{n}\left|\psi_{n, 2}^{(0)}\right\rangle,\left|\psi_{n, 4}^{(1)}\right\rangle=\frac{i m S}{2 B} \kappa_{n}\left|\psi_{n, 1}^{(0)}\right\rangle .
\end{aligned}
$$

Evaluating now the third order corrections in perturbation theory from Eq. (A5) they again turn out to be zero. This is also the case of the second order corrections to the eigenfunctions; i.e., from Eq. (A3) we get $\left|\psi_{n, i}^{(2)}\right\rangle=0 \forall n, i$. Thus we can calculate fourth order corrections to the energies from Eq. (A5) which are non-vanishing. Finally, we see that the fifth order correction again vanishes. In conclusion, our results for the perturbed spectrum, up to sixth order in perturbations is given by

$$
\begin{aligned}
\lambda_{n, 1} & =\lambda_{n, 3} \\
& =B\left[-2\left(n+\frac{1}{2}\right)+1+\frac{m^{2} S^{2}}{2 B^{2}}-\frac{m^{4} S^{4}}{8 B^{4}}+\mathcal{O}\left(\frac{m^{6} S^{6}}{B^{6}}\right)\right], \\
\lambda_{n, 2} & =\lambda_{n, 4} \\
& =B\left[-2\left(n+\frac{1}{2}\right)-1-\frac{m^{2} S^{2}}{2 B^{2}}+\frac{m^{4} S^{4}}{8 B^{4}}+\mathcal{O}\left(\frac{m^{6} S^{6}}{B^{6}}\right)\right] .
\end{aligned}
$$

Once we know the perturbed spectrum we can readily calculate the traces in Eq. (28). We will only perform the Dirac traces, but not the functional trace that is equivalent to the integration $\int d^{4} x$. Thus we obtain the result for the effective Lagrangian $w$ that as expected does not depend on $x$ :

$$
\begin{aligned}
2 w[V, S]= & 4 i \int_{0}^{\infty} \frac{d s}{s^{2}} e^{-i s\left(m^{2}-i \epsilon\right)}\left(\frac { B } { 8 \pi ^ { 2 } } \operatorname { c o s } \left\{s B \left[1+\frac{m^{2} S^{2}}{2 B^{2}}\right.\right.\right. \\
& \left.\left.-\frac{m^{4} S^{4}}{8 B^{4}}+\mathcal{O}\left(\frac{m^{6} S^{6}}{B^{6}}\right)\right]\right\} \\
& \left.\times \sum_{n} e^{i s[-2 B(n+1 / 2)]}-\frac{i}{(4 \pi)^{2} s}\right)
\end{aligned}
$$

Finally performing explicitly the addition of the series in $n$, we obtain

$$
\begin{aligned}
2 w[V, S]= & -\frac{4}{(4 \pi)^{2}} \int_{0}^{\infty} \frac{d s}{s^{2}} e^{-i s\left(m^{2}-i \epsilon\right)} \\
& +\left[\begin{array}{c}
\cos \left\{s B\left[1+\frac{m^{2} S^{2}}{2 B^{2}}-\frac{m^{4} S^{4}}{8 B^{4}}+\mathcal{O}\left(\frac{m^{6} S^{6}}{B^{6}}\right)\right]\right\} \\
\sin (s B)
\end{array}-\frac{1}{s}\right] .
\end{aligned}
$$


It can be seen that the result is purely real [29]; i.e., there is no particle production in the presence of constant magnetic and axial fields. In addition, the integral in $s$ is divergent in the ultraviolet limit $s \rightarrow 0$. As usual these divergences have to be removed by adding suitable counterterms. In order to obtain them, let us expand the part of the integrand in brackets around $s=0$; we have

$$
\begin{aligned}
& B \frac{\cos \left\{s B\left[1+\frac{m^{2} S^{2}}{2 B^{2}}-\frac{m^{4} S^{4}}{8 B^{4}}+\mathcal{O}\left(\frac{m^{6} S^{6}}{B^{6}}\right)\right]\right\}}{\sin (s B)}-\frac{1}{s} \\
& =B\left[\frac{1}{B s}-\frac{s B}{2}-\frac{s m^{2} S^{2}}{2 B}+\mathcal{O}\left(\frac{m^{6} S^{6}}{B^{6}}\right)\right]-\frac{1}{s}+\mathcal{O}\left(s^{3}\right) .
\end{aligned}
$$

The $\mathcal{O}\left(s^{3}\right)$ give rise to finite contributions when integrated. The first term is exactly cancelled by the $1 / s$ substraction. But we will have to include new counterterms proportional to $s\left(B^{2}+m^{2} S^{2}\right)$. Notice that these are exactly the same operators that we found in the perturbative calculation in Sec. III. In fact there is no contribution from $S^{4}$ operators due to an exact cancellation of the different quartic terms in the expansion in Eq. (41). Since the theory is renormalizable, it is necessary that the same kind of cancellation operates on the higher order terms on the right-hand side of the above expression.

The presence of the axial field is known that does not introduce any gauge anomaly in the electromagnetic current [30]; therefore the effective action will be gauge invariant. As a consequence it should be built out of scalar and gauge invariant functions. In our case, with constant magnetic and torsion fields, the only possibilities are $F_{\mu \nu} F^{\mu \nu}=2\left(\vec{B}^{2}\right.$ $\left.-\vec{E}^{2}\right)$ and $F_{\mu \nu}^{*} F^{\mu \nu}=(4 \vec{B} \cdot \vec{E})^{2}$. Since the second term vanishes in our case with constant magnetic or electric fields, the effective action is invariant under the transformation $B$ $\rightarrow-i E$ [29]. In this way we can convert our results for constant magnetic field to pure constant electric fields. Taking into account that now $\sin (s B) \rightarrow-i \sinh (s E)$ and $\cos \left\{s B\left[1+m^{2} S^{2} /\left(2 B^{2}\right)-m^{4} S^{4} /\left(8 B^{4}\right)\right]\right\} \rightarrow \cosh \left\{s E\left[1-m^{2} S^{2} /\right.\right.$ $\left.\left.\left(2 E^{2}\right)-m^{4} S^{4} /\left(8 E^{4}\right)\right]\right\}$ and integrating using the residues technique we can obtain the expression for the imaginary part of the effective Lagrangian:

$$
\begin{aligned}
p= & 2 \operatorname{Im} w[E, S]=\frac{1}{4 \pi^{3}} E^{2} \sum_{n=1}^{\infty} \frac{(-1)^{n}}{n^{2}} \\
& \times \cos \left\{n \pi\left[1-\frac{m^{2} S^{2}}{2 E^{2}}-\frac{m^{4} S^{4}}{8 E^{4}}+\mathcal{O}\left(\frac{m^{6} S^{6}}{E^{6}}\right)\right]\right\} e^{-m^{2} n \pi / E} .
\end{aligned}
$$

When $S=0$ we recover the original Schwinger result in Eq. (4). Notice that in the absence of electric field there is no particle production even with non-vanishing axial field. When the mass is zero, we recover the usual result; i.e., axial fields only contribute in the massive case. In Fig. 1 the upper

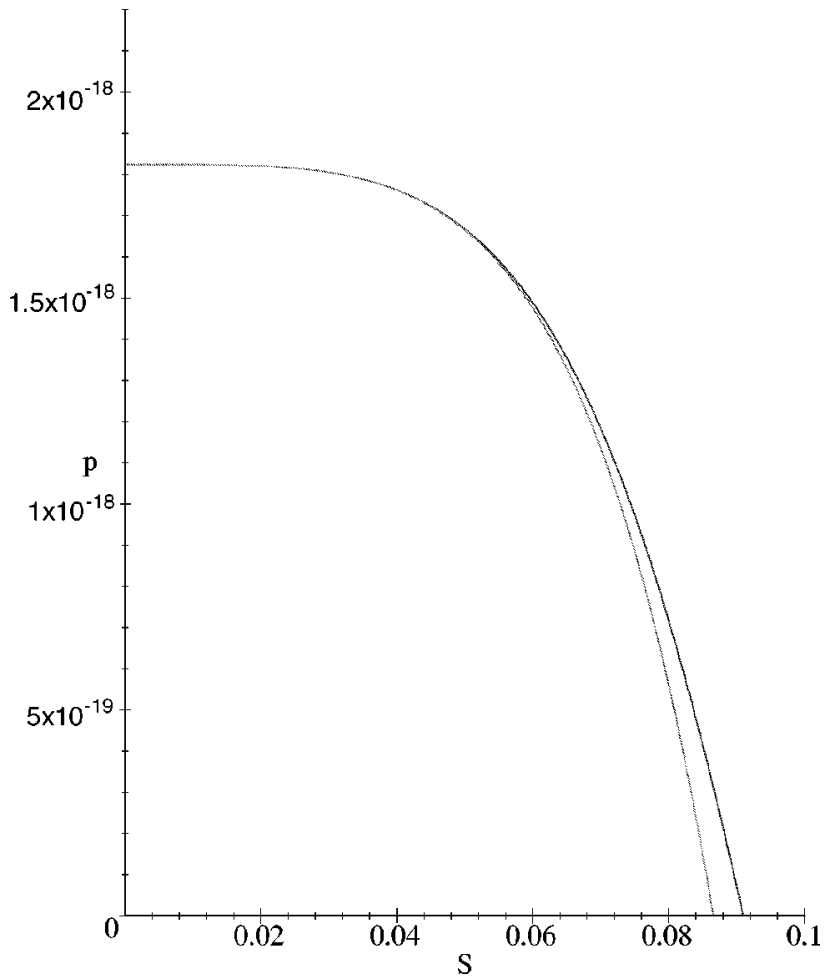

FIG. 1. Probability densities $p$ in units $m^{4}$ versus axial field $S$ in units $m$, for an electric field $E=0.1 \mathrm{~m}^{2}$. The upper curve represents the perturbative calculation up to sixth order. The lower curve is the resummation of the perturbative series estimated in the text.

curve represents the probability density $p$ as a function of $S$ for $E=0.1 \mathrm{~m}^{2}$; this value for the electric field ensures that the condition $S^{2}<E$ is satisfied for all the values of $S$ in the plot.

We can try to extend further the above result by means of the following observation. Since the only possible divergences in our model are those mentioned before, it is necessary that, when expanding the cos function in Eq. (41), the higher order terms in $S$ cancel; this implies

$$
s B^{2}\left(1+\frac{m^{2} S^{2}}{2 B^{2}}-\frac{m^{4} S^{4}}{8 B^{4}}+\cdots\right)^{2}=s\left(B^{2}+m^{2} S^{2}\right) .
$$

Therefore we can obtain the complete result for the effective action to all orders in perturbation theory in the limit $S^{2}$ $\ll B$. Performing the rotation to electric fields, the result is given by

$$
\begin{aligned}
p & =2 \operatorname{Im} w[E, S] \\
& =\frac{1}{4 \pi^{3}} E^{2} \sum_{n=1}^{\infty} \frac{(-1)^{n}}{n^{2}} \cos \left(n \pi \sqrt{1-\frac{m^{2} S^{2}}{E^{2}}}\right) e^{-m^{2} n \pi / E} .
\end{aligned}
$$

In Fig. 1, this probability is represented by the lower curve. We see that the effect of the axial field is to suppress particle production. Eventually it could make it to vanish. The point of vanishing $p$ should indicate the breakdown of the perturbative approximation since the probabilities should 
be positive. In order to check whether the perturbative calculation is valid up to the point in which $P$ vanishes, we will study the convergence of the perturbative series. In particular, there is a general result due to Kato [31] that states that if there are two non-negative constants $a$ and $b$ such that $\left\|H_{1}\right\| \leqslant a|| H_{0}|\psi\rangle||+b|\| \psi\rangle||$ for all $|\psi\rangle \in D\left(H_{0}\right)$ and if the operator $H_{1}$ is bounded, then the eigenvalues perturbative series is absolutely convergent if $2\left\|H_{1}\right\|<d$. With $d$ being the distance from $\lambda_{n, i}^{0}$ to the rest of the spectrum of $H_{0}$. In our case, we take $a=0$ and provided $\left\{\left|\psi_{n, i}\right\rangle\right\}$ is a complete set of eigenfunction we can expand $H_{1}|\psi\rangle$ $=H_{1} \Sigma_{n, i} C_{n, i}\left|\psi_{n, i}\right\rangle$. It is then easy to show that $\| H_{1}|\psi\rangle \|^{2}$ $=m^{2} S^{2} \Sigma_{n, i} C_{n, i}^{2}=m^{2} S^{2}$. Therefore if we take $b=m S$, since $H_{1}$ is bounded (it is constant) and in our case $d=2 B$, we have that the series is absolutely convergent for $m^{2} S^{2} /\left(2 B^{2}\right)<1 / 2$. Transforming to the electrostatic case, if we look at the plot, we can realize that for those particular values, the point in which the curve crosses the axis is in fact approximately signaling the breakdown of the perturbative series.

\section{CONCLUSIONS}

In this work we have studied the production of massive fermions from a classical vector and axial-vector background. Using a perturbative evaluation of the effective action we have obtained the contributions to the imaginary part of the effective action up to second order in the external fields. We have shown that in the reference frame for which $\vec{k}=0$, the production from purely spatial axial fields is suppressed with respect to that of the vector background. In addition, for purely temporal axial fields it is possible to create particles whereas this is not the case for vector fields.

In the particular case of a small constant axial field and a constant electric field, it is shown that a non-perturbative calculation can be carried out when those fields are orthogonal. In this case, it is shown that, in the massless limit, the axial field does not affect the production from the electric field. However, in the massive case, the presence of the axial background inhibits such production.

Finally, let us compare these result with the anisotropy damping phenomenon at the Planck era. As is well-known, the presence of small anisotropies in the early universe can be damped in a few Planck times due to the back reaction of the particles produced on the geometry. An interesting possibility is that a similar mechanism could take place in the presence of some primordial torsion field. In this case, since torsion can be generated by the intrinsic spin, this could happen when the fermions are produced in some configuration such that the total spin angular momentum of the system did not vanish. The use of effective action methods for fermions could be extended to the production of higher spin fields, such as gravitinos in a straightforward way. In addition, it is also interesting to study not only the particle production rate derived from the effective action, but also the spectra and angular distribution of fermions produced. This could be approached by means of the traditional Bogolyubov technique. Work is in progress in this direction [32].

\section{ACKNOWLEDGMENTS}

I thank Ed. Copeland and A. Dobado for useful suggestions. This work has been partially supported by the Ministerio de Educación y Ciencia (Spain) (CICYT AEN96-1634). The author also acknowledges support from SEUID-Royal Society.

\section{APPENDIX}

In this appendix we summarize the main formulas of the standard perturbation theory used in the text. Let us assume that the Hamiltonian of theory can be decomposed in

$$
H=H_{0}+H_{1} .
$$

As shown in the text, we denote by $\lambda_{n, i}^{(0)}$ and $\left|\psi_{n, i}^{(0)}\right\rangle$ the eigenvalues and eigenfunctions of $H_{0}$, which are assumed to be known. The unperturbed spectrum is assumed to be nondegenerated and discrete. The eigenfunctions and eigenvalues of the complete Hamiltonian $H$ are expanded in series: $\lambda_{n, i}=\Sigma_{p=0} \lambda_{n, i}^{(p)}$ and $\left|\psi_{n, i}\right\rangle=\Sigma_{p=0}\left|\psi_{n, i}^{(p)}\right\rangle$ where

$$
\lambda_{n, i}^{(p)}=\left\langle\psi_{n, i}^{(0)}\left|H_{1}\right| \psi_{n, i}^{(p-1)}\right\rangle
$$

and

$$
\left|\psi_{n, i}^{(p)}\right\rangle=S_{n, i}\left(H_{1}\left|\psi_{n, i}^{(p-1)}\right\rangle-\sum_{k=1}^{p-1} \lambda_{n, i}^{(k)}\left|\psi_{n, i}^{(p-k)}\right\rangle\right)
$$

with

$$
S_{n, i}=\sum_{j \neq i} \frac{\left|\psi_{n, j}^{(0)}\right\rangle\left\langle\psi_{n, j}^{(0)}\right|}{\lambda_{n, i}^{(0)}-\lambda_{n, j}^{(0)}} .
$$

Some simplified formulas for the lowest order terms in the spectrum are given by

$\lambda_{n, i}^{(1)}=\left\langle\psi_{n, i}^{(0)}\left|H_{1}\right| \psi_{n, i}^{(0)}\right\rangle$,

$\lambda_{n, i}^{(2)}=\sum_{j \neq i} \frac{\left|\left\langle\psi_{n, i}^{(0)}\left|H_{1}\right| \psi_{n, j}^{(0)}\right\rangle\right|^{2}}{\lambda_{n, i}^{(0)}-\lambda_{n, j}^{(0)}}$,

$\lambda_{n, i}^{(3)}=\left\langle\psi_{n, i}^{(1)}\left|H_{1}-\lambda_{n, i}^{(1)}\right| \psi_{n, i}^{(1)}\right\rangle$,

$\lambda_{n, i}^{(4)}=\left\langle\psi_{n, i}^{(1)}\left|H_{1}-\lambda_{n, i}^{(1)}\right| \psi_{n, i}^{(2)}\right\rangle-\lambda_{n, i}^{(2)}\left\langle\psi_{n, i}^{(1)} \mid \psi_{n, i}^{(1)}\right\rangle$,

$\lambda_{n, i}^{(5)}=\left\langle\psi_{n, i}^{(2)}\left|H_{1}-\lambda_{n, i}^{(1)}\right| \psi_{n, i}^{(2)}\right\rangle-2 \lambda_{n, i}^{(2)} \operatorname{Re}\left\langle\psi_{n, i}^{(1)} \mid \psi_{n, i}^{(2)}\right\rangle$

$-\lambda_{n, i}^{(3)}\left\langle\psi_{n, i}^{(1)} \mid \psi_{n, i}^{(1)}\right\rangle$. 
[1] N.D. Birrell and P.C.W. Davies, Quantum Fields in Curved Space (Cambridge University Press, Cambridge, England 1982).

[2] L. Parker, Phys. Rev. Lett. 21, 562 (1968); Phys. Rev. 183, 1057 (1969); in Asymptotic Structure of Space-Time, edited by F.P. Esposito and L. Witten (Plenum, New York, 1977).

[3] F. Cooper, J.M. Eisenberg, Y. Kluger, E. Mottola, and B. Svetisky, Phys. Rev. D 48, 190 (1993).

[4] J. Schwinger, Phys. Rev. 82, 664 (1951).

[5] M. Gasperini and M. Giovannini, Phys. Rev. D 47, 1519 (1993).

[6] J. Garriga and E. Verdaguer, Phys. Rev. D 39, 1072 (1989).

[7] E. Brézin and C. Itzykson, Phys. Rev. D 2, 1191 (1970).

[8] N. Narozhnyi and A. Nikishov, Sov. J. Nucl. Phys. 11, 596 (1970).

[9] Y.B. Zel'dovich and A.A. Starobinski, Pis'ma Zh. Éksp. Teor. Fiz. 26, 373 (1977) [JETP Lett. 26, 252 (1977)].

[10] L. Kofman, A. Linde, and A.A. Starobinski, Phys. Rev. D 56, 3258 (1997); Y. Shtanov, J. Traschen, and R. Branderberger, ibid. 51, 5438 (1995).

[11] P.B. Greene and L. Kofman, hep-ph/9807339.

[12] V.F. Mukhanov, H.A. Feldman, and R.H. Brandenberger, Phys. Rep. 215, 203 (1992).

[13] M. Gasperini, M. Giovannini, and G. Veneziano, Phys. Rev. Lett. 75, 3796 (1995).

[14] J.B. Hartle and B.L. Hu, Phys. Rev. D 20, 1772 (1979).

[15] A. Dobado and A.L. Maroto, gr-qc/9803076.

[16] P. van Nieuwenhuizen, Phys. Rep. 68, 189 (1981).
[17] M.B. Green, J.H. Schwarz, and E. Witten, Superstring Theory (Cambridge University Press, Cambridge, England, 1987).

[18] R.R. Metsaev and A.A. Tseytlin, Nucl. Phys. B293, 92 (1987).

[19] E.J. Copeland, A. Lahiri, and D. Wands, Phys. Rev. D 51, 1569 (1995); 50, 4868 (1994); J.D. Barrow and K.E. Kunze, ibid. 55, 623 (1997).

[20] A.L. Maroto and I.L. Shapiro, Phys. Lett. B 414, 34 (1997).

[21] E. Cartan, C. R. Hebd. Seances Acad. Sci. 174, 593 (1922).

[22] R. Utiyama, Phys. Rev. 101, 1597 (1956); T.W.B. Kibble, J. Math. Phys. 2, 212 (1961).

[23] I.L. Buchbinder, S.D. Odintsov, and I.L. Shapiro, Effective Action in Quantum Gravity (IOP, Bristol, 1992).

[24] A. Dobado and A.L. Maroto, hep-th/9712198.

[25] I.L. Buchbinder, S.D. Odintsov, and I.L. Shapiro, Phys. Lett. 162B, 92 (1985).

[26] C. Itzykson and J.B. Zuber, Quantum Field Theory (McGrawHill, New York, 1980).

[27] A. Galindo and P. Pascual, Quantum Mechanics (SpringerVerlag, Berlin, 1991).

[28] I.S. Gradshteyn and I.M. Ryzhik, Table of Integrals, Series and Products (Academic, New York, 1980).

[29] W. Dittrich and M. Reuter, Effective Lagrangians in Quantum Electrodynamics (Springer-Verlag, Berlin, 1985).

[30] A. Dobado and A.L. Maroto, Phys. Rev. D 54, 5185 (1996).

[31] T. Kato, Perturbation Theory for Linear Operators (SpringerVerlag, Berlin, 1980).

[32] A.L. Maroto and A. Mazumdar, hep-ph/9811288. 